\title{
Local environments of SNe Ic and Ic-BL
}

\author{
Jonatan Selsing ${ }^{1}$, Lise Christensen $^{1}$, Christina Thöne ${ }^{2}$ \\ and Maryam Modjaz ${ }^{3}$ \\ ${ }^{1}$ Dark Cosmology Centre, Niels Bohr Institute, University of Copenhagen, \\ Juliane Maries Vej 30, 2100 Copenhagen O, Denmark \\ email: jselsing@dark-cosmology.dk, lise@dark-cosmology.dk \\ ${ }^{2}$ Instituto de Astrofisica de Andalucia, \\ Glorieta de la Astronomia s/n, 18008 Granada, Spain \\ email: cthoene@iaa.es \\ ${ }^{3}$ Center for Cosmology and Particle Physics, New York University \\ Meyer Hall of Physics, 4 Washington Place, room 529, New York, NY 10003 \\ email: mmodjaz@nyu.edu
}

\begin{abstract}
We have observed the local explosion environments of a sample Type Ic and Type IcBL Supernove (SNe) selected from both targeted and non-targeted surveys using VLT/VIMOS in IFU-mode. It is believed that by probing the local surroundings of the parent stellar populations of these types of SNe, valuable information can be gained about the physical conditions, which affect the type of SNe produced. The different kinds of SNe produced are determined by the initial mass and metallicity of the stellar progenitor, as well as by the metallicity-dependent mass loss in the stellar winds at the end phase of their evolution and the interaction with a sufficiently close companion star.
\end{abstract}

Keywords. nuclear reactions, nucleosynthesis, abundances, (stars:) supernovae: general

\section{Project}

At the redshift of the galaxies we have selected, we spatially resolve regions approx. 250 pc across, comparable to the size of HII regions in local galaxies and using strong nebular emission lines as a proxy for the metal content of the stellar population, we can investigate if the conditions for the two types of SNe differ. The connection between long-duration gamma-ray bursts (GRBs) and broad-lined SNe Ic and the existence of SNe Ic-bl without observed GRBs raises the question of what distinguishes a GRB progenitor from that of an ordinary SN Ic-bl without a GRB, and this project will help with the elucidation of this. Moreover, from the HII region ages and stellar mass estimates, we examine the two suggested progenitor models for stripped SNe: single massive Wolf-Rayet (WR) stars with main-sequence masses of larger than 30 solar masses that have experienced mass loss during the main sequence and WR stages, vs. binaries from lower-mass He stars.

To answers this, we have taken data for 19 SN Ic and Ic-BL hosts using the IFU installed at VLT/VIMOS which gives spatially resolved spectra allowing diagnostics to be determined locally which for the redshift of the host corresponds to the physical sizes of the molecular clouds hosting the stellar progenitor population. We show in Fig. 1 a mosaic of the $\mathrm{H} \alpha$ emission determined from the VIMOS data superposed on the SDSS images of the host which we can translate into a star formation rate. Additionally using stellar population modeling, we can constrain the progenitor age and thereby the mass that will help discerning between the different types of progenitor stars.

For the metallicities determined locally we reproduce the trend seen earlier that Ic-BL prefer lower metallicity environments as compared to normal Ic. This is still preliminary very work and the values might chance slightly. A thing to note is that the $1-\sigma$ intervals 


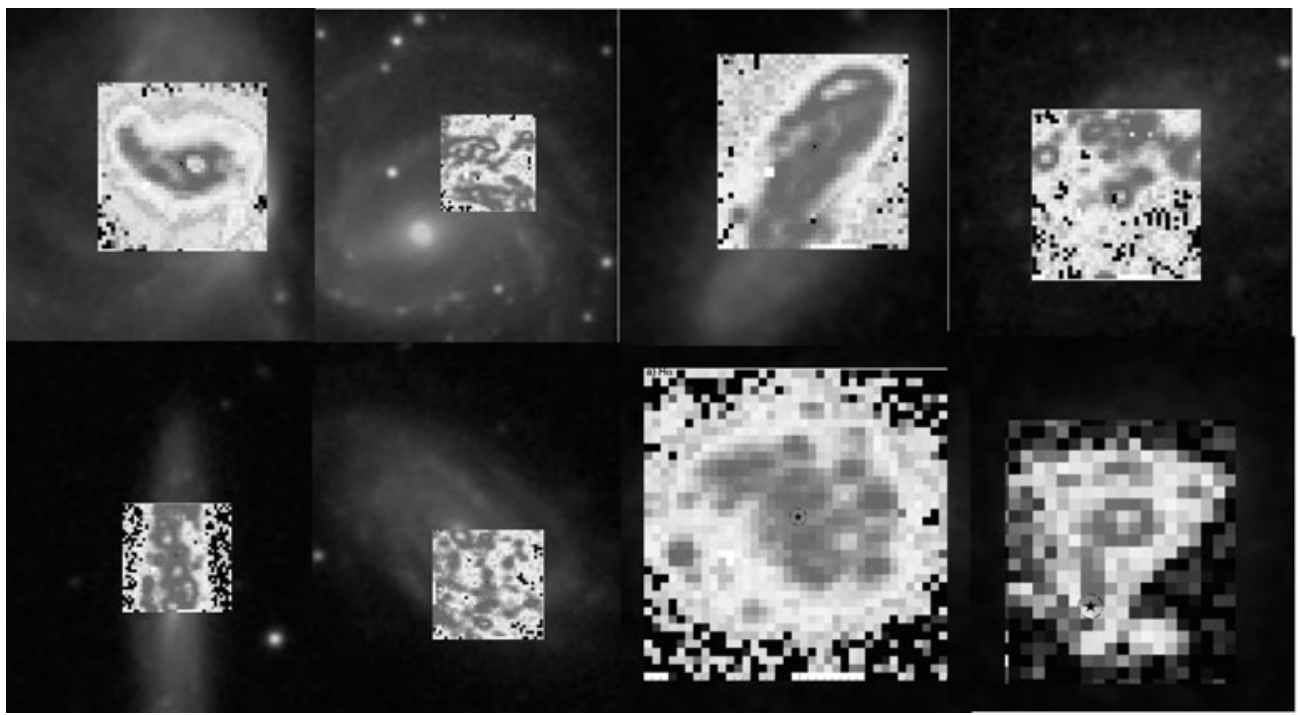

Figure 1. A mosaic showing 8 of the targeted SN Ic and Ic -BL host galaxies with the H $\alpha$ flux determined from the VIMOS observation superposed on the SDSS postage stamps. This is part of our sample, investigating the local properties of helium-poor SNe.

around the mean values are consistent within the errors, therefore pointing to a nonsignificant difference in the types of environments preferred.

\section{References}

Kuncarayakti et al. 2013, AJ, 146, 30

Graham, J. F. \& Fruchter, A. S. 2013, ApJ, 774, 119 